\title{
The Creative Use of Excel in Questionnaire Design and Results Acquisition
}

\author{
Jianli Han \\ School of Economics and Management, Zaozhuang University, Zaozhuang 277160, China, \\ 165698650@qq.com
}

\begin{abstract}
Taking the online shopping survey of college students as an example, this paper discusses the design of questionnaire and method for obtaining survey results based on Excel. Excel can be used to quickly design the questionnaire and read the questionnaire data. This method is simple, efficient and high accuracy. At the same time, using Excel can make the investigation effectively expand the sample size, thereby improving the accuracy and credibility of the findings.
\end{abstract}

Keywords: questionnaire; questionnaire design; results acquisition; Excel.

\section{巧用Excel进行调查问卷设计及结果获取}

\author{
韩建丽 \\ 疋庄学院经济与管理学院, 丰庄 中国
}

摘 要: 本文以 “大学生网络购物情况调查” 为例探讨了基于Excel的调查问卷设计和调查结 果的获取方法。利用EXCEL可以快速地设计问卷并读取调查问卷的数据, 该方法简单、高效、 准确性高, 同时不受调查样本数量的影响, 可以有效地扩大样本容量, 从而提高调查结果的 精确度和可信度。

关键词: 调查问卷; 问卷设计; 结果获取; Excel

\section{1. 前言}

统计调查是收集第一手资料进行研究的重要方法，而问卷调查又是其中应用最为广泛的 方法之一。传统的纸质问卷调查在存储、发放、回收、统计等环节会耗费大量的人力、物力 和财力, 且容易造成调研问卷的缺失。由于人力、物力和财力的限制, 调查样本的数量和广 度也会受到限制, 从而影响调查结果的精确度和可信度。随着信息技术的进步, 近年来网上 问卷调查因其调查成本低、速度快、隐匿性好、交互性强、结果可靠客观等优点得到快速发 展, 但往往要求调查者有专业的网络知识, 才能够在网上进行问卷的编辑和处理。其实, 网 络知识缺乏的人想进行统计调查并不困难, 巧用 Excel 就可以轻松地进行网上问卷调查、结 果获取和分析。本文仅以 “大学生网络购物情况调查” 为例探讨基于 Excel 的调查问卷设计 和调查结果的获取。

\section{2. 统计调查问卷设计}

中文篇名为请注意表格的格式，请注意表格的格式，请注意表格的格式，请注意表格的 格式, 请注意表格的格式, 请注意表格的格式, 请注意表格的格式, 请注意表格的格式, 请 注意表格的格式，请注意表格的格式。

2.1. 软件准备

Exce12007 及更高版本能够在不打开某个文件的情况下获取该文件中的数据, 为了便于 调查结果的获取, 本文的相关操作是在 Exce12010 中进行的。 
调查问卷的设计用到 Excel 中的 “开发工具” 选项卡，显示该选项卡的方法是：文件选项一自定义功能区，在右边 “主选项卡”一栏中的 “开发工具” 前打勾, 然后单击 “确定” 。 2.2. 调查问卷界面设计

新建 Exce1 工作簿, 将其命名为 “大学生网络购物问卷调查” , 并保存在 “大学生网购 调查” 文件夹下。在 Sheet1 工作表标签上双击, 将其重命名为 “调查问卷”。在工作表中输 入标题、说明、调查题目等，并设置相应的格式，如图 1 中所示（文本部分）。

\section{大学生网络购物情况调查}

亲爱的同学, 您好! 感谢您能抽出宝贵的时间参与此次调查。此 劳驾您据实、认真填写, 答案没有对错之分。我在此向您表示深深的

1. 受访者基本信息

A. 年级

B. 性别

C. 月消费水平

D. 高校所在省份

2. 您是否有过网络购物经历?

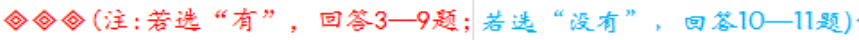

3. 近半年来您网络购物的次数是

4. 䜣半年来您网络购物的金硕是

5. 您通过网络购买过何类产品? (可多选)

$\square$ 炤装及鞋类田化妆品及饰品

6. 上述产品中您购买次数最多的是

其次是

再次是

图 1 调查问卷界面（部分截图）

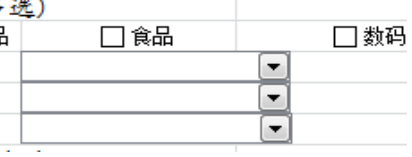

单选题选项的设置。图 1 中的 1-4 题均为单选题，其选项设置可以使用 “组合框” 控件 来实现。以第 3 题的选项为例, 绘制方法为: 单击开发工具-插入-表单控件-组合框，此时鼠 标变成小十字, 在 C10 单元格位置按住鼠标左键绘制组合框。其他题目的选项用同样的方法 设置，或者直接复制-粘贴已有的组合框控件。

多选题选项的设置。图 1 中的第 5 题为多选题，其选项设置可以使用 “复选框” 控件来 实现。以第 5 题的第 1 个选项为例, 绘制方法为: 单击开发工具-插入-表单控件-复选框, 此 时鼠标变成小十字, 在 B13 单元格位置按住鼠标左键绘制复选框, 并将其重命名为 “服装和 鞋类”。其他选项用同样的方法设置，或者直接复制-粘贴已有的选项并重命名。

排序题选项的设置。图 1 中的第 6 题为排序题，其每一位次选项的设置可视为一个单选 题，用 “组合框” 控件来实现，方法不再赘述。

2.3. 编码设置

为实现调查问卷界面上各选项的功能, 并为后续的统计分析做准备, 需要为各选项设置 编码。在 Sheet2 工作表标签上双击, 将其重命名为 “编码设置”。由于第 1 题中 “高校所在 省份” 的备选答案最多, 是包含港澳台地区在内的我国 34 个省区, 因此代码为 1 到 34 。具 体的编码设置情况如图 2 所示。设置编码后, 统计问卷结果时可以直接用相应的编码来代替 答案，如当某份问卷的被调查者年级为 “大三” 时，则使用编码 3 代替; 月消费水平是 500-799 元之间时，则使用编码 2 代替; 其他以此类推。

\begin{tabular}{|c|c|c|c|c|c|c|c|c|c|}
\hline 4 & A & B & C & D & E & $\mathrm{F}$ & G & $\mathrm{H}$ & I \\
\hline 1 & 代码 & 1年级 & 1 性别 & 1月消费水 & 1省份 & 2网购经历 & 3网购次数 & 4 网购金额 & 5 网购产品种类 \\
\hline 2 & 1 & 大一 & 男 & 499元以下 & 北京市（京 & 有 & 1-3次 & 199元以下 & 服装及鞋类 \\
\hline 3 & 2 & 大二 & 女 & $500-799$ & 天津市 (津 & 没有 & 4-6次 & 200-499元 & 化妆品及笛品 \\
\hline 4 & 3 & 大三 & & $800-1199$ & 上海市（沪） & & 7-10次 & 500-999元 & 食品 ～～～～ \\
\hline 5 & 4 & 大四 & & $1200-1599$ & 重庆市（渝） & & 11-19次 & $1000-1999$ 元 & 数码产品 \\
\hline 6 & 5 & 研究生 & & 1600以上 & 河北省（冀) & & 20次以上 & 2000元以上 & 点卡充值类 \\
\hline 7 & 6 & & & & 河南省（豫） & & & & 书籍及音像制品 \\
\hline 8 & 7 & & & & 云南省（云） & & & & 体育用品 \\
\hline 9 & 8 & & & & 辽宁省（辽） & & & & 其他 \\
\hline
\end{tabular}

图 2 编码设置界面（部分截图）

2. 4. 单份问卷调查结果的获取

为获取某份问卷的调查结果, 需建立自动显示调查结果的工作表。在 Sheet3 工作表标签 上双击, 将其重命名为 “调查结果”。根据设计的调查题目, 建立列变量, 如图 3 所示。通 
常每个单选题需要建立一个列变量, 排序题的每个位次需要建立一个列变量, 它们的结果均 为某个选项的代码; 而多选题的每个选项需要建立一个列变量, 当该选项被选中时, 值为 “TRUE”，当该选项未被选中时，值为 “FALSE”。
1 \begin{tabular}{ll|l|l|l|l|l} 
A & $B$ & $C$ & $D$ & $E$ & $F$ & $G$
\end{tabular} \begin{tabular}{l|l|l|l|l|l}
$\mathrm{H}$ & $\mathrm{I}$ & $\mathrm{J}$ & $\mathrm{P}$ & $\mathrm{Q}$ & $\mathrm{R}$
\end{tabular} 1-1年级 1-2性别 1-3月消: 1-4省份 2网购经 3网购次: 4网购金 5-1服装, 5-2化妆, 5-3食品 6-1最多 6-2其次 6-3再次， FALSE FALSE FALSE $\frac{1}{2}$

图 3 单份问卷调查结果界面（部分截图）

下面设置 “调查问卷”工作表各题目与 “调查结果”工作表各变量的链接。

1. 设置 “组合框” 控件与 “调查结果”工作表中单元格的链接。仍以“调查问卷”工作 表中第 3 题为例, 首先选中第 3 题后面的 “组合框” 控件, 然后右击, 选择 “设置控件格式” 命令, 在弹出的 “设置控件格式” 对话框中, 设置数据源区域为 “编码设置! $\$ G \$ 2: \$ G \$ 6$ ” (显 示的是几种可供选择的网购次数）、“单元格链接” 为 “调查结果! $\$ F \$ 2$ ”, 这样网购次数的 选择结果即在 “调查结果” 工作表中的 $\$ F \$ 2$ 单元格中呈现。其他 “组合框” 控件的设置方法 类似。

2. 设置 “复选框” 控件与 “调查结果”工作表中单元格的链接。仍以第 5 题的第 1 个选 项为例, 选中该控件, 右击, 选择 “设置控件格式” 命令, 在弹出的 “设置控件格式” 对话 框中, 设置单元格链接为 “调查结果! $\$ H \$ 2$ ”, 这样该选项的选择结果即在 “调查结果” 工作 表中的 $\$ H \$ 2$ 单元格中呈现。其他 “复选框” 控件的设置方法类似。

调查问卷设计完成后，为避免被受访者不慎修改调查结果，可以隐藏 “编码设置” 和 “调 查结果” 两个工作表, 方法为右键单击相应工作表标签, 在弹出的快捷菜单中选择 “隐藏” 命令。

\section{3. 问卷的发出与收回}

问卷的发出可以有多种形式, 比较常用的有: 利用电子邮件功能、利用 $\mathrm{QQ}$ 文件传输或者 群文件功能、利用百度云、微云、360 云盘等网盘功能等实现快捷方便的问卷发出。

问卷的收回可以通过前述发出问卷的各种方式收回问卷。但为了集中、方便地收回问卷， 调查者最好事先在调查问卷中说明问卷的收回时间和方式, 比如要求 “在 $\times$ 年 $\times$ 月 $\times$ 日之前 将调查问卷发送至某某电子邮箱”。等电子邮件都收到以后, 统一将调查问卷下载到同一文 件夹下。

\section{4. 问卷调查结果的获取及汇总}

现在调查问卷的结果散落在各个收回的调查问卷中，下一步要做的就是获取这些结果， 将其汇总到同一张工作表中，具体步骤如下:

1. 重命名收回的调查问卷。为便于获取各调查问卷的结果，需要将收回的问卷统一重命 名。在本例中, 将 “调查问卷” 文件夹中存放的 40 份调查问卷全部选中, 右键单击, 在弹出 的快捷菜单中选择 “重命名” 命令, 输入 “调查问卷”, 则各问卷被自动重命名为 “调查问 卷 (1)”、“调查问卷 $(2) ” 、 \cdots . .$. “调查问卷 $(40) ” 。$

2. 新建 Excel 工作簿, 将其命名为 “调查结果汇总” , 并保存在 “大学生网购调查” 文 件夹下，用于获取汇总的问卷调查结果。

3. 在 Sheet 1 工作表标签上双击, 将其重命名为 “汇总准备”。然后如图 4 所示, 在 A1 单元格输入 “问卷序号”, 在 A2: A41 单元格区域输入问卷的序号 1-40, 并将单份问卷调查 结果中第 1 行的列标题复制到 B1 及其右侧的区域。接着在 B2 单元格中输入公式: $="=$ " $\&$ INFO ("DIRECTORY") \&"调查问卷 $\backslash[$ 调查问卷 ("\& A2 \&").x1sx] 调查结果' ! A $\$ 2$ ", 并将 其复制到 B3: B41 单元格区域。 


\begin{tabular}{|c|c|c|c|c|c|c|c|c|c|c|c|c|c|c|}
\hline 4 & & B & C & D & $\mathrm{E}$ & $\mathrm{F}$ & G & $\mathrm{H}$ & I & $\mathrm{J}$ & $\mathrm{K}$ & $Q$ & $\mathrm{R}$ & $S$ \\
\hline 1 & 问卷序号 & 1-1年级 & 1-2性别 & -3月覑 & 1-4省你 & 2网购经 & 3网购行 & 4 网购金 & 5-1服浇 & 5-2化壮 & $5-3$ 鿖点 & 6-1最多 & 6-2其次 & 6-3再次 \\
\hline 2 & 1 & 1 & 1 & 2 & 12 & 2 & 0 & 0 & FALSE & FALSE & FALSE & 0 & 0 & 0 \\
\hline 3 & 2 & 1 & 2 & 1 & 12 & 2 & 0 & 0 & FALSE & FALSE & FALSE & 0 & 0 & 0 \\
\hline 4 & 3 & 1 & 1 & 3 & 14 & 1 & 4 & 4 & FALSE & FALSE & TRUE & 5 & 6 & 3 \\
\hline 5 & 4 & 1 & 2 & 2 & 6 & 2 & 0 & 0 & FALSE & FALSE & FALSE & 0 & 0 & 0 \\
\hline
\end{tabular}

图 4 汇总的问卷调查结果界面（部分截图）

下面解释一下 B2 单元格中的公式。该公式的作用是返回第 1 份问卷的第 1 个变量所在的 单元格地址。公式中 “INFO ("DIRECTORY”)” 的含义是返回当前文件的路径, 以保证本次研究 所在的文件夹 “大学生网购调查” 不管放在任何目录下，本文所给出的公式都能达到预期的 效果; 需要注意的是, 必须事先清空 Excel 软件的 “默认文件位置” , 方法是：单击文件选项-保存, 在弹出的对话框中删除 “默认文件位置” 中的文本, 然后单击 “确定”。公式中 的第一个 “调查问卷” 是所有问卷所在的文件夹名, 第二个 “调查问卷” 是本部分第 1 步中 重命名时输入的文件名, “调查结果” 是每份问卷的调查结果所在的工作表名, “A $\$ 2$ ” 是每 份问卷的结果的第一个变量所在的单元格地址（为了后续复制的方便必须写成这种形式）, 对于这些值, 读者将来可根据实际情况进行修改。

4. 在 Sheet2 工作表标签上双击, 将其重命名为 “结果汇总”。复制 “汇总准备”工作表, 然后使用 “粘贴-值” 命令将其粘贴到 “结果汇总” 工作表。接着选择 B2：B41 单元格区域, 使用 “替换” 命令，将 “=” 替换为 “=”，这样 “值” 就被替换为 “公式” ，这些单元格中 显示的就是所有样本调查结果的第 1 个变量的取值。最后选择 B2: B41 单元格区域, 待鼠标 变成小黑十字时, 拖动向右复制, 直到最后一个变量的位置, 这样所有样本的所有变量的取 值即获取完毕（本例中的部分结果如图 4 所示）。

需要说明的是, 被调查者未填写的内容, 在单份问卷的调查结果中显示为空值, 而这里 会显示为 “ 0 ”，不过这并不影响后续的统计分析，因为在 “编码设置” 里并没有 “ 0 ” 这个 代码, 这里刚好可以用它来代表缺失值。

总之，基于 Exce1 的统计调查问卷的设计、收发以及结果的获取非常简单实用，除 “组 合框” 和 “复选框” 控件的使用以及获取汇总的调查结果时的一个公式稍显复杂外，使用的 都是 Excel 的文本录入、复制、粘贴、重命名等常规操作, 问卷的收发借助电子邮件、QQ 等 常用工具, 因此调查者即使毫无基础也能轻松学会。该方法非常高效, 问卷收回后几步简单 的操作, 便可获得调查结果, 省去了繁琐的数据录入工作, 也避免了由此产生的数据录入错 误及其带来的不利后果。该方法还可以显著地增加样本的数量扩大调查的范围, 因为样本容 量增加时, 调查问卷设计工作不变, 结果获取也只是多填充了几个数据、多复制了几个公式 而已, 工作量基本不变; 电子邮件、QQ、网盘等工具的使用甚至允许调查者在全球范围内进 行调查, 由此可提高调查结果的精确度和可信度。

\section{References}

[1]. Chen Yonggui,Design and implementation of a questionnaire survey system based on Excel[J]. computer knowledge and technology, 2015 (6).p.55-57.

[2]. Wang Dongyan,Use Excel macro and VBA to implement convenient and quick investigation and statistics[J]. office automation, 2010 (11).p.44-47. 